American Journal of Infectious Diseases 6 (4): 107-109, 2010

ISSN 1553-6203

(C) 2010 Science Publications

\title{
A 28 Years Old Woman with Severe Headache and Few Episodes of Vomiting: A Case Report
}

\author{
${ }^{1}$ Ahmad Reza Nakhayi, ${ }^{1}$ Tasnim Eghbal Eftekhaari, ${ }^{1}$ Hossein Montazerghaem, \\ ${ }^{1}$ Seyedhamid Moosavy and ${ }^{2}$ Abdoulhossain Madani \\ ${ }^{1}$ Department of Infectious Disease, \\ ${ }^{2}$ Department of Epidemiology, \\ Infectious Disease Research Center, \\ Hormozgan University of Medical Sciences, Bandarabbas, Iran
}

\begin{abstract}
Problem statement: Cryptococcosis neoformans is commonest central nervous system infection in individuals infected with HIV. Most of the patients infected with Cryptococcus neoformans present with headache and visual changes. Approach: A 28 year old woman presented to Emergency Department (ED) with a chief complaint of headache, fever and vomiting and developed seizures during the first hour of admission. Firstly, diagnosed as cryptococcal meningitis, then, because of having a low White Blood Cell count (WBC) and low Total Lymphocyte Count (TLC) she was checked for HIV Ab and P24 Ag. Results: A diagnosis of HIV was made then her family members were checked for HIV and they were also HIV positive. Patient was discharged with a two month course of Fluconazole and after completion of antifungal treatment, Antiretroviral Treatment (HAART) was started. Conclusion: This case indicated that Cryptococcosis neoformans may develop in Immune-deficient patients, so complete laboratory workup and high index of suspicion to HIV should be under diagnosis.
\end{abstract}

Key words: HIV, episodes of vomiting, Cryptococcus neoformans, Total Lymphocyte Count (TLC)

\section{INTRODUCTION}

Case report: A 28 year old woman with severe headache and few episodes of vomiting was referred to our outpatient clinic and admitted in emergency ward. Her headache had been present since last two months beginning in frontal area extending to both parietal areas during her pregnancy for which she had been treated with amoxicillin diagnosed as sinusitis. Headache was persistent after her delivery. In her physical exam she was pale and had a generalized wasting. Her right eye was adducted, had diplopia and blurred vision. Pupils were reactive to light. No neck rigidity was seen in examination. Lymphadenopathy was absent and her lungs were clear with a normal breathing sound. Abdomen was soft with no organomegaly. Her extremities were also normal except for nail bed pallor. Presenting ED vital signs were a temperature of $40^{\circ} \mathrm{C}$ (axillary), blood pressure 95/60 $\mathrm{mm} \mathrm{Hg}$, pulse was filiform with a rate of $100 \mathrm{~min}^{-1}$ and respiratory rate was $20 \mathrm{~min}^{-1}$. Blood was sampled for diff count, Hb, ESR, wright, widal, 2ME, BUN, Cr and urine was sampled for analysis and culture. Laboratory evaluation revealed a serum white blood cell (WBC) count of $3.4 \times 10^{3}$ with $72 \%$ neutrophils, $25 \%$ lymphocytes, 3\% monocytes, a hemoglobin of 8.5 $\mathrm{gm} / \mathrm{dl}$ and platelets $88 \mathrm{k} /$ cumm. Peripheral blood smear showed hypochromia, ovalocytosis, elliptocytosis, microcytosis, schistocytosis, spherocytosis and tear drop RBC. While her basic electrolytes were unremarkable except for serum potassium which was 2 , the liver function panel revealed a total bilirubin of 0.8 $\mathrm{mg} \mathrm{dL} \mathrm{L}^{-1}$, alkaline phosphatase 100 units $\mathrm{L}^{-1}$, SGOT (AST) 40 units $\mathrm{L}^{-1}$, SGPT (ALT) 13 units $\mathrm{L}^{-1}$, ALP 147 and LDH 314. Urinalysis had gross hematuria and pyuria and urine was positive for nitrite. Wright, widal and 2ME were not remarkable. Sputum and CSF were negative for AFB and culture. The patient's condition aggravated during the first hour of admission, she had an episode of focal seizures and her level of consciousness decreased. She was admitted in ICU and her seizures were treated with diazepam $10 \mathrm{mg}$ IV immediate (stat) dose and as needed (p.r.n.), phenytoin $750 \mathrm{mg}$ IV infusion in 250cc normal saline and valproic acid $300 \mathrm{mg}$ twice daily. Chest $\mathrm{x}$ ray was not remarkable and abdominal sonography was normal.

Corresponding Author: Abdoulhossain Madani, Department of Epidemiology, Infectious Disease Research Center, Hormozgan University of Medical Sciences,Bandarabbas, Iran 
Brain computerized tomography scan was performed to rule out any contraindications of lumbar puncture (LP). Brain had mild cortical edema and no focal lesions were present. LP was performed and cerebrospinal fluid (CSF) was sampled for cell count, protein and glucose level, adenosine deaminase (ADA) and gram stain. Infectious disease specialist was consulted. CSF revealed 370 white blood cells with $53 \%$ lymphocytes, 0 monocytes $47 \%$ polymorphonuclear cells, low glucose at $37 \mathrm{mg} \mathrm{dL}^{-1}$ and high protein at $1000 \mathrm{mg}$ $\mathrm{dL}^{-1}$. Gram stain was negative and empirical treatment was started with antituberculous regimens (four regimen treatment with isoniazid, rifampicin, ethambutol and pyrazinamide) because of high prevalence of tuberculosis in south of Iran. ADA was 0.5 and LP was performed again for Indian ink stain. Indian ink stain was positive for Cryptococcus neoformans. According to this finding and persistence of fever, anti-tuberculosis agents were discontinued and antifungal treatment was started with Amphotericin B $35 \mathrm{mg}$ IV infusion/day in D5\%; because of unavailability of Flucytosine in this area, Fluconazole was substituted and administered $400 \mathrm{mg}$ daily. Valproic acid and phenytoin were discontinued. Because of low WBC count (3500) and a total lymphocyte level of 850 and fungal meningitis, HIV Ab and P24 Ag were checked and were positive. Patient was discharged with a two month course of Fluconazole and after completion of antifungal treatment, antiretroviral treatment (HAART) was started. Following a detailed and in-depth history revealed that her husband was an intravenous drug user (IDU) and resided outside Iran having unprotected sex. Her husband and children were checked for HIV and were positive. Consent was obtained from the patient to report this case anonymously.

\section{RESULTS AND DISCUSSION}

Headache being a symptom is seen in most patients presenting to ER. Chronic headaches are often a sign of chronic meningitis, which is often caused by infectious, inflammatory, neoplastic causes and toxic exposures. Cryptococcus is common and fatal in immunocompromised patients (Cheung et al., 2003; Hong et al., 2007) and being a major cause of fungal meningitis (especially in patients with AIDS) (Cheung et al., 2003; Ginsberg and Kidd, 2008), has been the AIDS defining illness for $60 \%$ of HIV-infected patients in whom it is diagnosed (Coyle, 1999).As an opportunistic T cell modulated mycosis, Cryptococcosis is an important disease in patients with a weak or suppressed immune system (Ginsberg and Kidd, 2008).
Cryptococcus is the fourth most common source of lifethreatening infection in AIDS patients after infections of cytomegalovirus, Pneumocystis carinii and Mycobacterium avium intracellulare (Hong et al., 2007). A study has reported that approximately $60 \%$ of headache presentation in patients with AIDS headache is due to cryptococcal meningitis or presumed bacterial sinusitis (Shih et al., 2000).

Globally, it is estimated that approximately 957,900 cases of cryptococcal meningitis occur each year, resulting in more than 600,000 deaths (Sar et al., 2004). Neurological dysfunction as the first manifestation of AIDS has been found in 10-20\% of symptomatic HIV infections (Botha and Wessels, 1999). In most cases neurologic involvement in patients with AIDS is due to infection with Mycobacterium tuberculosis and Cryptococcus neoformans and they account for $75 \%$ cases of meningitis; because of their frequency and morbidity, they should always be considered first in evaluation of a headache in a patient with AIDS but only $1.9 \%$ of cases initially present with cryptococcal infection (Hong et al., 2007). Patient described in this case had a rare etiology of a common presentation to ED. Classic meningitic findings, such as neck rigidity, are absent in $75 \%$ of cases The most common presentation of cryptococcosis is a subacute meningitis or meningoencephalitis with fever, malaise and headache (Shih et al., 2000). Visual changes have been reported and most of visual changes in HIV infected patients are attributable to Cryptococcus meningitis (Hong et al., 2007), as well as new onset seizure activity.

Clinical disease is rarely fulminant; in these cases the patient may present with coma and rapid progression to death (Coyle, 1999). Clues to the diagnosis in this case included an atypical headache (sub acute), high grade fever and new onset seizures. Computed tomography of the brain is normal in $50 \%$ of proven cases; however, a focal neurological exam and abnormalities on brain CT or MRI have both been linked to a poorer prognosis (Lakshmi et al., 2007) but this patient had no focal lesions except for mild cortical edema. The CSF WBC count is typically low $(<50$ microL ${ }^{-1}$ ) with a mononuclear predominance and the protein and glucose concentrations are usually only slightly abnormal (Coyle, 1999) but in our case CSF WBC count was high $\left(350\right.$ microL $\left.^{-1}\right)$ with a lymphocytic predominance (53\%), which was favorable for aseptic meningitis and normal gram stain of CSF suggested a probability of TB meningitis and normal Zeehl Neilsen staining for TB, indicated for Indian ink staining which was positive for yeast cells. CSF staining with India ink will show the typical 
encapsulated yeast forms in approximately $75 \%$ of patients (Coyle, 1999). This patient had a low WBC count $\left(3500\right.$ microL $^{-1}$ ) and a low total lymphocyte count (850) and presence of a fungal meningitis in addition to decreased total lymphocyte count guided us to check for HIV status, regarding this fact that cryptococcal meningitis is seen in $89.3 \%$ of patients with TLC less than 200 (Leite et al., 2004). Although number of people infected with HIV in Hormozgan province (in south of Iran) was 927 with 54 new cases till end of 2009 in a population of 1450000, but HIV infection is not suspected in many cases and is only checked in people having high risk behaviors. Precise prevalence of fungal infections in HIV infected patients are not known which may be due to under reporting of these infections. Patients with chronic headache and fatigue should be screened for possible aseptic meningitis and detection of fungal infections may improve in patients (whether immunocompetent or immunocompromised) suspected of meningitis if CSF fluid was sent for Indian Ink stain besides gram stain. A study has shown that immunosuppressed patients present with less typical clinical symptoms of meningitis (Lakshmi et al., 2007). Our patient presented with sinusitis and headache. She was not suspected and diagnosed for meningitis until she developed seizures. Delayed diagnosis is an important issue with this disease and has reported to increase morbidity and mortality (Juhi et al., 2009) and undiagnosed HIV in a pregnant woman, caused birth of an infected newborn. Diagnosis of one patient with HIV and checking the HIV status of her family members, resulted in diagnosis of 3 more HIV patients. As number of Persons Living with HIV/AIDS (PLWAs) increases, caring for them is a new rising problem. Adequate knowledge and positive attitude are important factors in providing better care for a patient (Mahin et al., 2010).

\section{CONCLUSION}

Clinicians should be informed that fungal meningitis should be suspected in patients with chronic headache and fatigue; high index of suspicion and full laboratory workup is required for more survival and diagnosing hidden cases of HIV.

\section{ACKNOWLEDGEMENT}

Authors wish to thank Dr. Reza Kiani and Dr. Abdullah Moosavi for their cooperation in preparing this report.

Conflict of interest: The authors declare that they have no conflict of interests.

\section{REFERENCES}

Botha, R.J. and E. Wessels, 1999. Cryptococcal meningitis in an HIV negative patient with systemic sarcoidosis. J. Clin. Pathol., 52: 928-930. DOI: 10.1136/jcp.52.12.928

Cheung, A., R. Rachlis and S.L. Shumak, 2003. A cryptic cause of cryptococcal meningitis. Canadian Med. Assoc. J., $168.451-452$. http://www.researchgate.net/publication/10895510 _A_cryptic_cause_of_cryptococcal_meningitis

Coyle, P.K., 1999. Overview of acute and chronic meningitis. Neurol. Clin., 17: 691-710. DOI: 10.1016/S0733-8619(05)70162-6

Ginsberg, L. and D. Kidd, 2008. Chronic and recurrent meningitis. Pract. Neurol., 8: 348-361. DOI: 10.1136/jnnp.2008.157396

Hong, Y.J., S. Jung, J.Y. Kim, S.B. Kwon and K.B. Song et al., 2007. Complete binocular blindness as the first manifestation of HIV-related cryptococcal meningitis. J. Clin. Neurol., 3: 212-214. DOI: 10.3988/jcn.2007.3.4.212

Juhi, T., M. BibhaBati, B. Aradhana, L. Poonam and D. Vinita et al., 2009. Cryptococcal Meningitis in a tertiary care hospital. Jpn. J. Med. Mycol., 50: 9599. DOI: $10.3314 /$ jjmm.50.095

Lakshmi, V., T. Sudha, V.D. Teja and P. Umabala, 2007. Prevalence of central nervous system cryptococcosis in human immunodeficiency virus reactive hospitalized patients. Ind. J. Med. Microbiol., 25: 146-149. DOI: 10.4103/02550857.32723

Leite, A.G., J.E. Vidal, F.B. Filho, S.R. Nogueira, A.C. Oliveira, 2004. Cerebral infarction related to cryptococcal meningitis in an HIV-infected patient: Case report and literature review. Brazilian J. Infect. Dis., 8: 175-179. DOI: 10.1590/S141386702004000200008

Mahin, J.M., D. Parivash, A.H. Madani, A. Azadeh and A. Zinab et al., 2010. Knowledge and attitude of persons living with HIV+/AIDS. Am. J. Infect. Dis., 6: 70-74. DOI: 10.3844/ajidsp.2010.70.74

Sar, B., D. Monchy, M. Vann, C. Keo, J.L. Sarthou, Y. Buisson, 2004. Increasing in vitro resistance to fluconazole in Cryptococcus neoformans Cambodian isolates: April 2000 to March 2002. J. Antimicrobial Chemotherapy, 54: 563-565. DOI: 10.1093/jac/dkh361

Shih, C.C., Y.C. Chen, S.C. Chang, K.T. Luh and W.C. Hsieh, 2000. Cryptococcal meningitis in nonHIV-infected patients. Q. J. Med., 93: 245-251. DOI: 10.1093/qjmed/93.4.245 